\title{
An Epigenetic Hypothesis for Human Brain Laterality, Handedness, and Psychosis Development
}

\author{
A.J.S. KLAR \\ Gene Regulation and Chromosome Biology Laboratory, National Cancer Institute at Frederick, \\ Frederick, Maryland 21702-1201
}

Mendelian genetics deals with heredity of variation through generations, and it does not directly concern itself with cellular differentiation required for development in higher multicellular organisms. Indeed, in the 1940s, developmental biologists, believing that genes were not important for development, went their separate ways from geneticists. How could the multiple arrays of cell types be generated when all cells of an organism possess the same set of genes questioning the importance of genetics to development? They thought something other than genes must be important for development. Only after the discovery of the phenomenon of gene regulation in Escherichia coli (Jacob and Monod 1961) did these fields begin to converge. Questions still remain unanswered about how development occurs. What controls the expressions of genes that regulate development with such remarkable precision such that different organs develop only at specific locations on the body? Thus far, the major paradigm for developmental gene regulation comprises the "morphogen-gradient model" (Brown and Wolpert 1990) that remains to be experimentally verified. For example, it is not known what controls the development of human brain laterality whereby left and right hemispheres structurally and functionally differ from one another. Because there is an unexplained partial association of human left- versus right-hand-use preference with the hemisphere of the brain that develops language, handedness and brain laterality development must be somehow related. Furthermore, an excess of left-handedness is associated with psychiatric diseases of schizophrenia and bipolar disorders; anomalies of brain laterality development may be relevant for disease causation. In this broad overview, we will discuss possible connections between the seemingly unrelated fields of cell-type determination in fission yeast and brain development in humans. We will summarize results of studies that suggest that the traits of brain laterality, handedness, and psychosis development result from an embryonic asymmetric cell division. A mitotic cell genetic ("mitogenetics") model postulates that a Watson versus Crick DNA strand-specific imprinting of a chromosome and its patterned segregation produces differentiated daughter cells and that psychosis results from genetics, without a conventional Mendelian gene mutation in families carrying chromosome 11 rearrangements.

\section{COMPLEX CORRELATION OF BRAIN HEMISPHERIC LATERALITY WITH HAND-USE PREFERENCE}

The brain contains left and right hemispheres, and often they are functionally nonequivalent. The one that processes the language, logic, and some math functions is called the "dominant" hemisphere, while the one that deals with things such as spatial perception, intuition, and creativity is called the "automatic" hemisphere (Klar 1999). The functional specialization is associated with development of structural differences in specific regions of the brain, such as the Broca's area that processes language cognition. Compared to the left/right-axis specification of visceral organs such as heart, lungs, liver, and stomach in vertebrates (Klar 1994), human brain hemispheric structural differences are relatively subtle, although prominent differences lie with the distribution of cognitive functions. What is fascinating is that $97 \%$ of individuals who prefer to use their right hand (right-handers, RH) for unimanual tasks, such as handwriting, develop a dominant hemisphere in the left side and the balance process language in the right or both hemispheres (Coren 1992). Thus, RH preference is highly correlated with brain laterality. The situation with left-handers and ambidextrous individuals, collectively termed non-righthanders $(\mathrm{NRH})$, is rather unclear but more interesting because in them the handedness trait and brain laterality are not correlated. Thus, there is a very clear association between these traits in most RH, but not in NRH. The reason and the significance of this complex association have remained a key unanswered question in the fields of brain development and psychology. Because of such association, psychologists have conducted all studies for defining the etiology of handedness. As nearly all RH develop a lateralized brain such that the dominant hemisphere develops on the left side of the brain, clearly brain laterality must be biologically/genetically specified. Had it not been genetically determined, there should have been random left- versus right-sided distribution of specialized hemispheres, as may be the case with NRH (Klar 1996). The mechanism for developing differentiated hemispheres remains unknown, but the basis for the complex association between these traits is becoming clearer. As a way to directly define the biological basis of brain later- 
ality development, many studies in the past have been designed to define the etiology of its indirect measure, i.e., of handedness preference.

\section{HANDEDNESS ETIOLOGY}

The causes of hand-use preference have been debated for centuries. Often this preference is considered to be a complex trait where several genes contribute, along with the culturally taught behavior, to affect one's hand practice. Three main arguments have been advanced against a strictly genetic etiology. First, nearly one-half of the children of NRH $x$ NRH cross are RH (Rife 1940). Second, in certain cultures the left-hand use is discouraged, so the hand preference can be culturally influenced. Third, and the pastiest problem, is that $18 \%$ of monozygotic twins, each co-twin possessing identical genetic constitutions, are discordant — one "lefty" and one "righty" - for handedness (Rife 1940). Because of these observations, hand preference is proposed in many studies to be a culturally taught behavior. Others posit brain damage during birth or a sudden surge of testosterone levels during pregnancy to influence one's handedness (for review, see Coren 1992). Such psychology models are difficult to test as they fail to make verifiable predictions. Instead, three one-locus models proposed random (50\% RH: $50 \% \mathrm{NRH}$ ) handedness of individuals carrying the nonfunctional allele. Two of these models propose additive effects of two alleles of a locus (Annett 1985; McManus 1991), while the more recent "random-recessive model" proposes a fully penetrant and dominant RGHT1 (for right-handedness) allele and a recessive $r$ (for random) allele (Klar 1996). Results of several relatively recent tests of the random-recessive model are summarized below.

First, the value of $7.6 \% \mathrm{NRH}$ born to $\mathrm{RH} \times \mathrm{RH}$ parents (Rife 1940) is predicted from the calculated 61\% RGHT1: $39 \% r$ allele frequency of RH individuals. This allele frequency was derived from the result of $19.6 \% \mathrm{NRH}$ born to RH x NRH parents (Klar 1996). Second, both RH and $\mathrm{NRH}$ born to $\mathrm{NRH} \times \mathrm{NRH}$ are expected from the random handedness of $r / r$ homozygotes (Annett 1985; McManus 1991; Klar 1996). Third, RH (whose both parents were $\mathrm{NRH}) \times$ standard $\mathrm{RH}$ produce increased proportion of $\mathrm{NRH}$ progeny, a result similar to the $\mathrm{RH} \times \mathrm{NRH}$ cross and different from that of the standard $\mathrm{RH} \times \mathrm{RH}$ cross (Klar 1996). Fourth, RH (twin of a discordant pair) $\times$ RH crosses produced a higher proportion of NRH children, similar to the NRH twin and the standard NRH when they are married with standard, unselected RH (Klar 2003). Thus, the genotype of discordant twins must be similar to that of NRH. Fifth, and the most decisive, finding comprises the recently discovered partial association of handedness with the direction of perital hair-whorl rotation found on the top of human head. Over $95 \%$ of individuals support a single whorl that rotates clockwise or counterclockwise. Moreover, a majority (91.6\%) of individuals in the general public carry clockwise hair whorls. Clearly, the direction of hair-whorl rotation is genetically determined. The most telling result is that NRH develop random hair-whorl orientation. Confirming this finding, individuals chosen only because of their counterclockwise swirls were equally divided between RH and NRH. Thus, the genetics that cause one to become RH must also cause the development of a clockwise hair whorl, and the genetics that cause NRH lead to randomness in hairwhorl orientation. Therefore, because of the association of handedness to brain laterality, we conclude that the RGHT1 allele causes the coupled development of dominant hemisphere on the left side of the brain, $\mathrm{RH}$ preference, and clockwise hair rotation. Those with the $r / r$ constitution develop these traits, but the traits are uncoupled from each other and are distributed randomly to the left versus right side of the body. We therefore propose that the counterclockwise whorl orientation signifies the $r / r$ genotype. This phenotype is much easier and definitive to score than handedness, and can be exploited to study the contribution of genetics of brain laterality development to other behavioral traits. For example, a 3.6-fold excess of counterclockwise whorl rotation is found in homosexual men (A.J.S. Klar, unpubl.). In the general public, only 9-11\% of persons are NRH (Rife 1940). Curiously, the value of NRH goes up 3-fold in patients suffering from major psychiatric diseases of schizophrenia and bipolaraffective disorders (Boklage 1977). Explaining this association is the main reason for deciphering the handedness etiology that in turn may shed light on psychosis etiology.

\section{PSYCHOSES ETIOLOGY REMAINS ENIGMATIC}

Schizophrenia is characterized by the inability of patients to differentiate between real and imaginary images and voices, suffering delusions and hallucinations, and the inability to function socially. Bipolar disease patients fluctuate between bouts of manic depression and high activity called mania. There is, however, considerable overlap in the symptoms of both diseases, including suicidal behavior, so that several studies have suggested a common etiology for both diseases. Both disorders are highly debilitating and remain incurable. Each disease affects about $1 \%$ of the population worldwide. Family, adoption, and twins studies point to genetic factors but, thus far, unambiguous identification of the important susceptibility genes has not been accomplished (for review, see Kennedy et al. 2003). A large number of studies of genome-wide scans produced significant evidence for linkage to many regions of the genome, but no result has been convincingly replicated. The lack of replication has been frequently attributed to the small number of patients investigated. However, a recent pooling of the data of large pedigrees from several studies failed to find significant linkage to chromosome regions for both disorders (Lewis et al. 2003; Segurado et al. 2003). Despite that, consensus of the field is that these are multifactor or "complex" traits and that they result from the combined effects of multiple genetic and environmental factors that interact with each other (Kennedy et al. 2003). As the consensus is based on negative results of not finding major disease-causing loci, the scientific basis for the consensus was recently questioned (Klar 2002). The best 
guess at present is that diseases result from anomalies of brain development as patients have less lateralized brain hemispheres, compared with healthy controls (Crow 1990; DeLisi et al. 1997). Significantly, NRH single borns and handedness-discordant twins also exhibit reduced brain hemisphere laterality, although they are not diseased (DeLisi et al. 1997; Geschwind et al. 2002). As noted above, psychotic patients are three times more likely to be NRH as compared with controls; it has been suggested that the genetics causing NRH may be a disease-predisposing factor (Boklage 1977; Annett 1985; Crow 1990; McManus 1991; Klar 1999). However, the exact cause is unknown; genetic factors have not been identified; and at present the cause of psychosis remains one of the most challenging problems in all of biology. Because of its importance for human health, it is an area of research vigorously pursued by a large number of investigators. Finding the cause of handedness may shed light on the cause of psychosis. Even so, identification of the handedness-determining gene is unlikely to be the end of the story, since the next great challenge facing us will be to determine how would the RGHT1 gene specify hemispheric laterality. Thinking ahead, we can imagine that the RGHT1 gene may control brain laterality development by promoting an asymmetric cell division during embryogenesis. The primary basis of asymmetric cell division at the single cell level is best understood by studies of a model system of the fission yeast. Could such a mechanism, combined with other speculations concerning diploid genome, explain handedness, brain laterality and psychosis development?

\section{"DIFFERENTIATED" WATSON VERSUS CRICK CHAIN INHERITANCE OF THE PARENTAL CHROMOSOME DIFFERENTIATES FISSION YEAST CELLS}

Schizosaccharomyces pombe is a haploid yeast whose rod-shaped cells grow by equational division. Its cells exist in two cell/sex types, called "P" (for "plus") and "M" (for "minus"), respectively controlled by the alternate alleles of the mating-type locus (mat1). An 40-kb matingtype region located in chromosome 2 also contains closely linked silent copies of the mating-type information in mat2-P and mat3- $M$ "donor loci." The $\mathrm{P}$ and $\mathrm{M}$ cell types interconvert by a transposition/substitution reaction in which a copy of either the mat 2 or mat 3 locus is unidirectionally transferred to the expressed mat1 locus (for review, see Klar 2001). The mat1 switching is highly efficient; however, all cells do not exhibit a uniform ability to switch. A remarkable pattern of switching is observed in mitotic pedigrees such that only one of four granddaughter cells of a newly switched cell switches mating type (Miyata and Miyata 1981). As shown in Figure 1, the Pu ("u" for unswitchable) cell nearly always produces one daughter $(\mathrm{Pu})$ like itself, while the other daughter is advanced in its developmental program as it acquires switching competence (Ps, "s" for switchable). The Ps cell divides to produce one of the switched daughters to M type. This pattern is called the "one-in-four" or

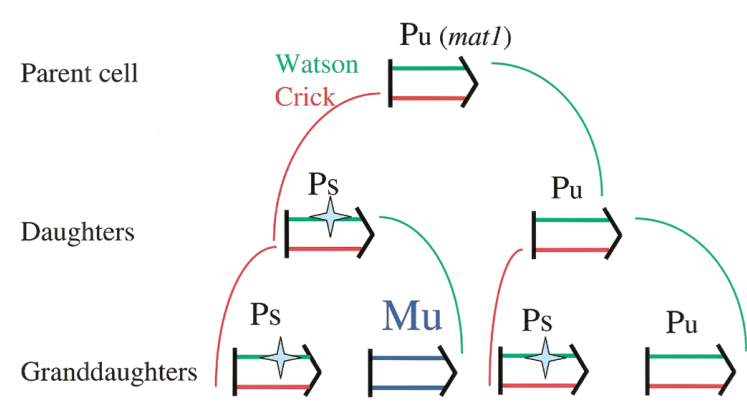

Figure 1. The strand-segregation mechanism explains the switching pattern of fission yeast cells. The Pu ("u" for unswitchable) parent cell mostly produces one Ps ("s" for switchable), and the other is always a Pu daughter cell. This is because the Ps daughter inherits the parental-Watson-strandcontaining chromatid (indicated in green) that is imprinted (imprint indicated by star on the upper strand), and $\mathrm{Pu}$ inherits a nonimprinted parental-Crick-strand-containing chromatid (in red). The imprint is installed at mat1 during DNA replication by a strand-, site-, and sequence-specific DNA alteration. In this mechanism, only the arbitrarily named Watson strand is imprinted. Next, when the imprinted strand is used as a template for replication (i.e., in the Ps cell), a transient double-stranded chromatid break results that initiates recombination interaction with mat $2-P$ or $m a t 3-M$ donor loci, resulting in mat 1 switching. The sister of the switched cell is most often switching-competent as indicated with a Ps designation. Thus, two consecutive asymmetric cell divisions result in switching of only one-in-four granddaughters of a Pu cell. The DNA sequence of the mat1-M allele is distinguished in blue, as it differs from that of the mat1$P$ allele. The newly switched cell is of Mu type and its progeny will also switch by the one-in-four pattern.

the "granddaughter" switching rule. Thus, only one (Ps in Fig. 1) of the two daughter cells of an unswitchable cell $(\mathrm{Pu})$ is competent to produce a single switched daughter cell in the following generation.

Further pedigree analysis produced another "consecutive" switching rule: The sister of the recently switched cell is switching-competent (Egel and Eie 1987; Klar 1990). The M cells likewise switch to P type by following the same rules. Notably, this switching process follows the stem cell-like pattern found in some systems of cellular differentiation in eukaryotes. The key observation is that each cell division produces developmentally nonequivalent daughter cells. It is remarkable that this fungus follows a precise developmental program that takes two generations to complete even though it is a single-celled organism! Are we witnessing an evolution of a biological process that may be crucial for evolving multicellular organisms? Understanding the mechanism of an asymmetric cell division in any system may help us explain cellular differentiation required for development in higher eukaryotes.

The asymmetric cell division is explained with the strand-segregation model (Fig. 1) (Klar 1987, 1990). According to the model, sister cells differ simply because one inherits the "imprinted" Watson strand from the parental chromosome while the sister inherits the nonimprintable Crick strand. "Imprint" is defined as an epige- 
netically inherited, reversible and nonmutational alteration of DNA. Attached proteins, modified bases including ribose nucleotides, or a nick in one strand of the DNA were hypothesized for the biochemical nature of the imprint (Klar 1987, 1990). Replication of the imprinted strand results in a transient double-stranded break (DSB) of the chromatid, and the break initiates recombination of mat 1 with donor loci by the DSB repair mechanism. Meiotic mat1 gene conversion analysis showed that the imprint is chromosomally borne, linked to mat1, and installed in one generation, but it is used in the next one for mat1 conversion (Klar and Bonaduce 1993). Two other tests of the model predicted that cells engineered to carry inverted matl duplication should produce developmentally equivalent daughter cells since both daughters will inherit identical sequences, one through the parental Watson strand and the other through the Crick strand. Consequently, each daughter should inherit one (never both) imprinted, switching-competent cassette that should exhibit DSB; and, second, both daughters should produce one switched and one unchanged daughter of their own. Thus, only one cassette should show DSB break in a cell; second, unlike the one-in-four rule followed by wild-type cells, two (cousins)-in-four granddaughters should switch. Both predictions were experimentally verified, establishing the strand-segregation model (Klar 1987, 1990). Recent studies have further supported the details of the model. For example, the imprint is installed only on the strand that is replicated by the lagging-strand replication complex (Dalgaard and Klar 1999) and it may be a site-specific nick (Arcangioli 1998) or an alkali-labile modified base (Dalgaard and Klar 1999), such as a ribose nucleotide moiety (Vengrova and Dalgaard 2004). The key lessons learned from yeast studies is that developmental asymmetry of sister cells derives from Watson and Crick DNA chains being complementary and not identical (Watson and Crick 1953) and that differentiated chromatids result from DNA replication because of strand-specific imprinting. Since the model is well established by several genetic and molecular studies, hereafter it will be referred to as the strand-segregation mechanism. Another important lesson we learned is that epigenetically established states of gene expression causing silencing of mat 2 and mat 3 are passed on to progeny as conventional Mendelian/chromosomal markers, both in mitosis and in meiosis (Grewal and Klar 1996). It is clear that sometimes the Mendelian gene is composed of DNA plus an epigenetic moiety, and both of them are faithfully replicated during chromosome replication as Mendelian variations (Klar 1998). In principle, such mechanisms for asymmetric cell division and stable states of gene expression discovered in yeast can be imagined to control the development of human brain hemispheric laterality.

\section{THE STRAND-SPECIFIC IMPRINTING AND SEGREGATION (SSIS) MODEL FOR BRAIN LATERALITY AND PSYCHOSIS DEVELOPMENT}

The yeast cells are haploid, but human cells are diploid and therefore contain two homologs of each chromo- some. To apply the strand-segregation mechanism to humans, one has to invoke coordination of imprinting and patterned segregation of differentiated chromatids (hence parental strands) of both copies of a chromosome to daughter cells during mitosis to affect an asymmetric cell division. Such a model was proposed recently (Klar 1999, 2002, 2004). Genetic evidence supporting it is covered in primary papers to which the readers should refer for details. Only salient features will be highlighted here with the aim to place those studies in a wider context.

The key proposal of the SSIS model is to make differentiated sister chromatids by expressing a hypothetical gene named $\mathrm{DOH} 1$ in one, say, parental Watson strandcontaining chromatid, but epigenetically silencing it in the other chromatid (Fig. 2). This occurs during DNA replication of both copies of a specific chromosome in a certain cell division when brain laterality development is initiated. In addition, another hypothetical SEG site must exist elsewhere in the chromosome used to nonrandomly place sister chromatids at the metaphase plate such that a parental Watson with Watson and Crick with Crick pattern of segregation will result. The trans-acting factor encoded by the RGHT1 gene may directly or indirectly interact with the $S E G$ site to effect patterned segregation of DNA chains to progeny cells. Thus, one daughter cell will inherit $\mathrm{DOH} 1$ "epialleles" that are transcriptionally active $(O N)$, while the other daughter inherits unexpressed $(O F F)$ epialleles. Such a single asymmetric cell division in embryogenesis is hypothesized to the development of brain hemispheres in which language is processed in the left hemisphere and the right one becomes an automatic hemisphere. It should be noted that epigenetic controls for gene regulation come in many forms: The epigenetic control may exhibit a parent-of-origin effect; one of the two homologs of a chromosome may be stably inactivated such as with the $\mathrm{X}$ chromosome; the genes, such as mat-donor loci in fission yeast, and genomic parasites, such as retroelements, are permanently silenced. The SSIS model adds to this list of epigenetic controls by postulating the evolution of somatic strand-specific imprinting to cause asymmetric cell division. It is simply a developmental biology model postulating strand-specific imprinting and patterned-segregation mechanism in embryogenesis for regulating the expression of a developmentally important gene. The model implies that perhaps an important function of the epigenetic phenomenon concerns somatic cell differentiation. The model does not specify the molecular nature of the epigenetic event, but its nature is likely to involve an assembly of heterochromatin structure analogous to the silencing mechanism of fission yeast mat loci (Grewal and Klar 1996; Klar 1998).

As no other hypothesis explains the biology behind brain laterality development, we are forced to consider new ideas. The SSIS model remains formally an abstract model, as we do not know which cell division and which chromosome are involved; whether the $\mathrm{DOH} 1$ gene actually exists, what function it performs, and the mechanisms of strand-specific imprinting and segregation remain unknown. Despite these unknowns, a test of the model is provided by studies of rearrangements of the relevant chromosome where the $S E G$ site is dissociated 


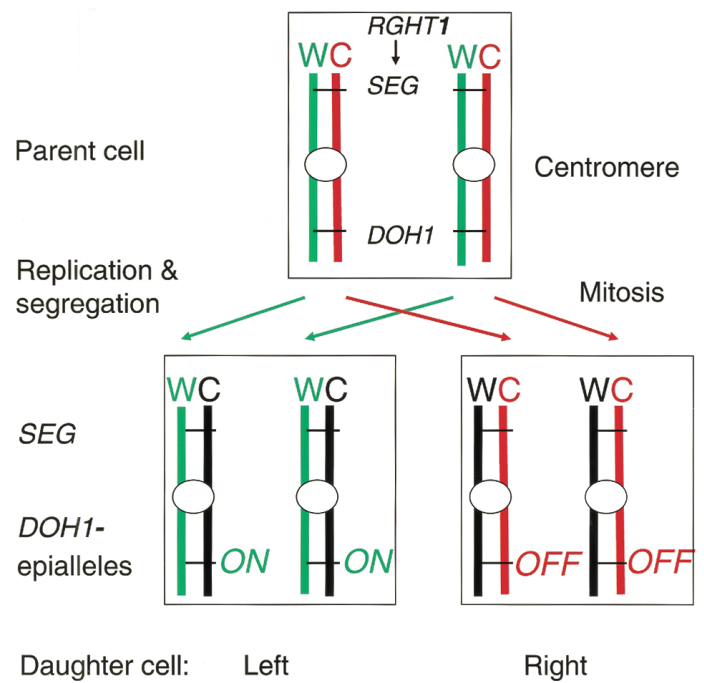

Figure 2. The somatic strand-specific imprinting/segregation model - the SSIS model - advanced to produce nonequivalent daughter cells in mitosis during human embryonic development. The model postulates production of developmentally nonequivalent sister chromatids at a specific cell division. The hypothetical DOHI (for Dominant hemisphere-specifying) gene is transcriptionally active $(O N)$ in parental chromosome-derived Watson (W) strand-containing chromatid, and it remains silenced $(O F F)$ by an epigenetic mechanism in the parental Crick (C) strand-containing sister chromatid. Second, a linked hypothetical $S E G$ (segregation) site resides elsewhere in the chromosome to effect patterned distribution of differentiated chromatids to specific ("leftward" versus "rightward" placed with respect to dorsal/ventral axis of the embryo) daughter cells in the embryo. The $S E G$ element may or may not coincide with the centromere. Third, RGHT1 (for right-handedness) gene-encoded factor, acts on the $S E G$ site directly, or indirectly, for patterned distribution of sister chromatids of both homologs to specific daughter cells. The chains are color-coded to indicate their distribution to specific daughter cells. An asymmetric segregation occurs so that both parental W chains (green) go to one daughter cell, and both Cs (red) to the other; the newly synthesized chains are indicated in black. In summary, differentiated daughter cells result by inheriting $\mathrm{DOH} 1$ epialleles and, after subsequent growth, result in development of differentiated brain hemispheres.

from the $\mathrm{DOH} 1$ gene owing to a chromosome translocation in one of the homologs. In this situation, the $\mathrm{DOHI}$ epialleles in the translocation chromosome will be randomly distributed, as the chromosome lacks an $S E G$ site, but the epialleles in the wild-type chromosome will be distributed in a patterned fashion. Consequently, one-half of the translocation heterozygotes should produce a healthy, lateralized brain (Fig. 2). In the remaining onehalf, both daughters will be become equivalent, as both will inherit $O N / O F F D O H 1$ epialleles, resulting in development of symmetrical hemispheres and possibly causing psychosis. This explanation was recently advanced to explain the result of 18 diseased among 36 heterozygous translocation carriers of a large Scottish pedigree with the $\mathrm{t}(1 \mathrm{q} 42 ; 11 \mathrm{q} 14)$ translocation (Evans et al. 2001). Interestingly, of the 18 , nine were diseased with schizophrenia and the remainder with the bipolar disorder. Thus, both of these disorders can be considered as manifestations of the same genetic etiology. However, results of a single translocation do not rule out the conventional explanation in which the translocation produces a dominant diseasecausing mutation. Indeed, two overlapping genes on chromosome 1, named DISC (for "disrupted in schizophrenia") 1 and 2 , carry mutations in the translocation. According to the conventional explanation, incomplete penetrance of these mutations has been postulated to explain disease occurrence in some and not in other translocation heterozygotes (Evans et al. 2001).

To identify the relevant chromosome according to the SSIS model, be it 1 or 11 , and to test whether a specific gene is mutated by the $\mathrm{t}(1 \mathrm{q} 42 ; 11 \mathrm{q} 14)$, studies of other translocations may be useful. A recent study searched the Medline database with the query "psychosis and translocation." In addition to studies describing the aforementioned translocation, the search found several papers describing other translocations that also partially cosegregate with psychosis (Klar 2004): t(17q24;11q23), only one case found (Hoshi 1999); t(6q14;11q25), two diseased and two healthy (Holland and Gosden 1990); and $\mathrm{t}(9 \mathrm{p} 24 ; 11 \mathrm{q} 23)$, six diseased and five healthy (Baysal et al. 1998). Based on these findings, the following conclusions were reached (Klar 2004): First, only chromosome 11 is relevant to psychosis development, as it is a common participant in all the translocations; second, as only one-half of translocation carriers are diseased, the feature of patterned segregation of chromosome 11 strands is supported; and, third, the translocations lie at three far apart regions spanning $\sim 40 \%$ of the chromosome, so it is unlikely that a single gene is mutated by disparate translocations. Also, three groups working on these translocations failed to find linkage of the translocation junction region with inheritance of the disease in other families without chromosomal rearrangements (summarized in Klar 2004). In one case the nearest gene is located $299 \mathrm{~kb}$ away from both junction regions. Moreover, the linkage to the chromosome 1q arm where $\mathrm{DISCl}$ and 2 reside is also not supported by a multicenter study of a large number of patients (Levinson et al. 2002). Therefore, we assume that genetic alterations at the translocation junction regions are not causing disease. Together, these findings suggest that disease occurs in translocation carriers by disruption of patterned distribution of $\mathrm{DOH} 1$ epialleles, that there is no conventional Mendelian gene mutation, and that it is clearly a genetically caused disease, as it is partially cosegregating with translocations. This analysis does not imply that all diseased individuals should carry chromosome 11 rearrangements. As noted above, the RGHT1 gene controls the development of brain laterality, presumably through its interaction with the $S E G$ site to effect patterned segregation mechanism (Fig. 2); and combined with the observation that psychotic patients are three times more likely to be NRH as compared with healthy controls, nearly all psychotically diseased persons in the general public may be that way because of their $r / r$ genetic constitution. Clearly, only a minority of NRH is diseased; it remains to be tested whether $r / r$ genetic constitution alone or in combination with other variations causes the disease. It should be stated that at present there is no experimental 
evidence demonstrating the hypothesized $r / r$ genotype as the disease-predisposing factor. At present, we should only consider results with translocations as the best evidence favoring genetic etiology.

\section{PATTERNED DNA CHAIN SEGREGATION PROBABLY OCCURS IN MICE}

For the phenomenon of strand-specific imprinting in a diploid organism to be biologically useful, the SSIS model also requires evolution of the process of patterned chromatid segregation. As yeast is a haploid and singlecelled organism, no need can be perceived for the existence of patterned segregation process. After it was discovered that sister chromatids of fission yeast are nonequivalent simply because of inheriting parental Watson and Crick chains (Klar 1987, 1990), the process of patterned segregation had to be invoked if such a mechanism were to evolve for evolving multicellular eukaryotes. It is generally assumed that identical daughter chromosomes are produced by DNA replication; therefore, experimentally testing the patterned segregation possibility seems unwarranted. Would some unrelated study fortuitously shed light on this possibility?

Liu et al. (2002) investigated whether the Cre-loxP recombination system could be used for genetic alterations of mouse chromosomes. They placed the recombination cassettes at allelic sites near the centromeres of two chromosomes, and then they induced recombination by introducing the plasmid expressing the recombinase enzyme in embryonic stem (ES) cells. Site-specific recombination was observed for both chromosomes. A marker distal to the crossover point was maintained in the heterozygous constitution in some and homozygosis occurred in other recombinants of a chromosome. This outcome is expected from random segregation of DNA chains after recombination in the $G_{2}$ phase of the cell cycle. Curiously, all 432 recombinants exhibited homozygosis of a distal marker located near the tip of chromosome 7. To explain this unusual result, the authors suggested that the process of recombination might have so placed recombinant chromatids at the metaphase plate, causing them to segregate away from each other. An alternate possibility was suggested where patterned segregation of Watson with Watson and Crick with Crick occurs (Klar 2004), as indicated by the SSIS model (Fig. 2). Following this suggestion, one mouse chromosome undergoes random segregation but chromosome 7 follows the patterned chain distribution. It should be pointed out that if all chromosomes were to undergo patterned segregation at each cell division, the process would not be useful as a gene regulation control for development. For it to be useful, different sets of chromosomes should follow patterned distribution in different cell types. It was then noted that mouse chromosome 7 , which presumably undergoes patterned segregation, displays a $36 \%$ sequence synteny with human chromosome 11 implicated in psychosis (Klar 2004). It would be interesting to determine the strand segregation pattern of human chromosome 11 as well.

To facilitate the patterned segregation process, it would

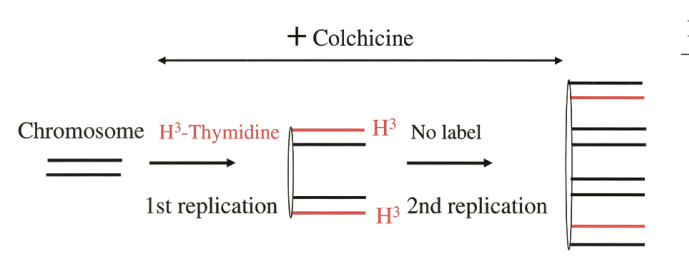

Result
$\mathrm{H}^{3}$

$\mathrm{H}^{3}$

Figure 3. Nonrandom distribution of newly synthesized DNA chains to daughter chromatids in diplochromosomes of endoreduplicated human blood cells (Schwarzacher and Schnedl 1966). The cells were labeled with $\mathrm{H}^{3}$-thymidine in the first of the two DNA replication cycles. Because of the colchicine treatment, a second round of replication occurs without cell division to produce diplochromosomes because daughter chromatids fail to separate at mitosis. Autoradiograph of the metaphase arrested diplochromosomes showed label restricted to the outside chromatids of each chromosome.

be helpful if the location of DNA chains at the centromere were fixed. Sister chromatids sometimes fail to separate at mitosis when cells are treated with the colchicine drug. Following another round of replication, four chromatids (i.e., eight DNA chains) remain paired in a structure called "diplochromosome." When human blood cultures were labeled with $\mathrm{H}^{3}$-thymidine in the first replication cycle, followed by another without the label, remarkably, the diplochromosomes had the outer chromatids labeled (Fig. 3 ), and not the inner ones (Schwarzacher and Schnedl 1966). Thus, DNA chains must hold fixed positions on the centromere and the newly synthesized chains are segregated to the "outer" chromatids. The importance of this result should now be viewed in the context of the SSIS model. This experiment also showed that mitotic recombination is rare, as most chromosomes did not show crossover events. Clearly, much remains to be done to test details of the SSIS model. Unfortunately, testing details of the SSIS model in humans is nearly impossible. Perhaps a useful approach will be to study hypothesized processes in experimentally amenable model systems, such as yeast, mice, and Drosophila. Interestingly, the fly has been recently shown to develop brain hemispheric asymmetry and it is implicated in long-term memory (Pascual et al. 2004).

\section{CONCLUSIONS}

The most fundamental unanswered question in developmental biology is how the diversity of cell types develops from a single cell, such as a fertilized egg. It is often thought that cellular differentiation must be controlled by cytoplasm factors that are unevenly expressed or distributed to daughter cells. Instead, nonequivalent daughter chromatids are produced in yeast, causing daughter cells to become different by the strand-segregation mechanism. It is taken for granted that two brain hemispheres develop differently from each other in healthy individuals, but the biology behind that development remains unknown and unapproachable. The SSIS model suggests a mechanism to effect differential hemisphere-specific gene regulation. It is suggested that brain laterality results from Watson and Crick chains being inherently different 
in acquiring an epigenetic event that regulates developmentally important gene(s). Accordingly, psychosis results when the proposed normally nonrandom segregation of DNA chains is randomized by chromosome 11 translocations. The most novel aspect of this interpretation is that the disorder is not due to conventional gene mutations or malregulation of genes due to epigenetic alterations at the breakpoint region, yet it is clearly a genetically caused disease. The model neatly explains a perplexing problem of how each of three different translocations causes disease only in one-half of the heterozygous rearrangement carriers, thus explaining the genetic oddity as they cause both dominant and recessive mutations. The view of genetic cause without Mendelian mutation is not at variance with the Watson-Crick base pairing or Mendel's rules. Mendelian genetics apply to inheritance through meiosis, but mitotic epigenetic control mechanisms strictly concern the use of genetic material in mitosis for accomplishing development even though all cells of the organism contain the same set of Mendel's genes. This mitogenetic concept implies that Watson and Crick chains can carry additional heritable (epi)genetic information to be used for somatic cell differentiation and that somatic interchromosomal and sister chromatid recombination would be deleterious as it would disrupt distribution of epialleles. For example, such unwanted mitotic recombinant events may lead to loss of heterozygosity for imprinted genes causing cancer development. Such a requirement may be one of the reasons for mitotic recombination rates to be low as compared with meiotic rates. Furthermore, the SSIS model also implies that for regulating developmentally important genes such as Hox genes, their linkage and order will be conserved during speciation. Overall, this view may provide a new paradigm for deciphering the cause of highly debilitating diseases and for explaining development in general. The brain tissue and hair whorls are derived from an ectoderm layer of cells where this mechanism may have evolved for brain laterality development. The same mechanism for visceral left/right axis determination in vertebrates has been also proposed for organs derived from mesoderm and endoderm tissues (Klar 1994). It is possible that a single chromosome dictates left/right-axis specification of neuronal and visceral organs by functioning in different layers of embryonic cells. At present, the prominent paradigm for development comprises the morphogen-gradient model, but it remains nontestable in most biological systems because the model fails to make refutable predictions. Cell-lineagebased strand-segregation mechanisms, those exploiting somatically imposed epigenetic mechanisms of gene controls, should be considered as another way to accomplish development.

\section{ACKNOWLEDGMENTS}

The author thanks colleagues of the Gene Regulation and Chromosome Biology Laboratory for discussions over many years that helped sharpen ideas presented here. The National Cancer Institute funded the work.

\section{REFERENCES}

Annett M. 1985. Left, right, hand and brain: The right shift theory. Lawrence Erlbaum, London.

Arcangioli B. 1998. A site- and strand-specific DNA break confers asymmetric switching potential in fission yeast. EMBOJ. 17: 4503

Baysal B.E., Potkin S.G., Farr J.E., Higgins M.J., Korcz J., Gollin S.M., James M.R., Evans G.A., and Richard C.W., III. 1998. Bipolar affective disorder partially cosegregates with a balanced $\mathrm{t}(9 ; 11)(\mathrm{p} 24 ; \mathrm{q} 23.1)$ chromosomal translocation in a small pedigree. Am. J. Med. Genet. 81: 81 .

Boklage C.E. 1977. Schizophrenia, brain asymmetry development, and twinning: Cellular relationship with etiological and possible prognosis implications. Biol. Psychol. 12: 19.

Brown N.A. and Wolpert L. 1990. The development of handedness in left/right asymmetry. Development 109: 1.

Coren S. 1992. Left-hander syndrome-The causes and consequences of left-handedness. Macmillan, New York.

Crow T.J. 1990. The continuum of psychosis and its genetic origins. The sixty-fifth Maudsley Lecture. Br. J. Psychiatry 156: 788.

Dalgaard J.Z. and Klar A.J. 1999. Orientation of DNA replication establishes mating-type switching pattern in $S$. pombe. Nature 400: 181.

DeLisi L.E., Sakuma M., Kushner M., Finer D.L., Hoff A.L., and Crow T.J. 1997. Anomalous cerebral asymmetry and language processing in schizophrenia. Schizophr. Bull. 23: 255.

Egel R. and Eie E. 1987. Cell lineage asymmetry for Schizosaccharomyces pombe: Unilateral transmission of a high-frequency state of mating-type switching in diploid pedigrees. Curr. Genet. 3: 5.

Evans K.L., Muir W.J., Blackwood D.H., and Porteous D.J. 2001. Nuts and bolts of psychiatric genetics: Building on the Human Genome Project. Trends Genet. 17: 35.

Geschwind D.H., Miller B.L., DeCarli C., and Carmelli D. 2002. Heritability of lobar brain volumes in twins supports genetic models of cerebral laterality and handedness. Proc. Natl. Acad. Sci. 99: 3176.

Grewal S.I. and Klar A.J. 1996. Chromosomal inheritance of epigenetic states in fission yeast during mitosis and meiosis. Cell 86: 95.

Holland T. and Gosden C. 1990. A balanced chromosomal translocation partially co-segregating with psychotic illness in a family. Psychiatry Res. 32: 1 .

Hoshi S. 1999. Acute promyelocytic leukemia with $\mathrm{t}(11 ; 17)(\mathrm{q} 23 ; \mathrm{q} 21)$. Rinsho Ketsueki 40: 119.

Jacob F. and Monod J. 1961. Genetic regulatory mechanisms in the synthesis of proteins. J. Mol. Biol. 3: 318.

Kennedy J.L., Farrer L.A., Andreasen N.C., Mayeux R., and St. George-Hyslop P. 2003. The genetics of adult-onset neuropsychiatric disease: Complexities and conundra? Science 302: 822 .

Klar A.J.S. 1987. Differentiated parental DNA strands confer developmental asymmetry on daughter cells in fission yeast. Nature 326: 466.

. 1990. The developmental fate of fission yeast cells is determined by the pattern of inheritance of parental and grandparental DNA strands. EMBO J. 9: 1407.

-1994. A model for specification of the left-right axis in vertebrates. Trends Genet. 10: 391.

. 1996. A single locus, RGHT, specifies preference for hand utilization in humans. Cold Spring Harbor Symp. Quant. Biol. 61: 59.

. 1998. Propagating epigenetic states through meiosis: Where Mendel's gene is more than a DNA moiety. Trends Genet. 14: 299.

. 1999. Genetic models for handedness, brain lateralization, schizophrenia, and manic depression. Schizophr. Res. 39: 207.

2001. Differentiated parental DNA chain causes stem cell pattern of cell-type switching in Schizosaccharomyces pombe. In Stem cell biology (ed. D.R. Marshak et al.), p. 17. Cold Spring Harbor Laboratory Press, Cold Spring Harbor, New York. 
2002. The chromosome 1;11 translocation provides the best evidence supporting genetic etiology for schizophrenia and bipolar affective disorders. Genetics 160: 1745.

2003. Human handedness and scalp hair-whorl direction develop from a common genetic mechanism. Genetics 165: 269.

2004. A genetic mechanism implicates chromosome 11 in schizophrenia and bipolar diseases. Genetics 167: 1833.

Klar A.J. and Bonaduce M.J. 1993. The mechanism of fission yeast mating-type interconversion: Evidence for two types of epigenetically inherited chromosomal imprinted events. Cold Spring Harbor Symp. Quant. Biol. 58: 457.

Levinson D.F., Holmans P.A., Laurent C., Riley B., Pulver A.E., Gejman P.V., Schwab S.G., Williams N.M., Owen M.J., Wildenauer D.B., et al. 2002. No major schizophrenia locus detected on chromosome 1q in a large multicenter sample. Science 296: 739.

Lewis C.M., Levinson D.F., Wise L.H., DeLisi L.E., Straub R.E., Hovatta I., Williams N.M., Schwab S.G., Pulver A.E., Faraone S.V., et al. 2003. Genome scan meta-analysis of schizophrenia and bipolar disorder. II. Schizophrenia. Am. J. Hum. Genet. 73: 34

Liu P., Jenkins N.A., and Copeland N.G. 2002. Efficient CreloxP-induced mitotic recombination in mouse embryonic stem cells. Nat. Genet. 30: 66.
McManus I.C. 1991. The inheritance of left-handedness. In Biological asymmetry and handedness (ed. J. Marsh), p. 251. Wiley, Chichester, United Kingdom.

Miyata H. and Miyata M. 1981. Mode of conjugation in homothallic cells of Schizosaccharomyces pombe. J. Gen. Appl. Microbiol. 27: 365.

Pascual A., Huang K.L., Neveu J., and Preat T. 2004. Neuroanatomy: Brain asymmetry and long-term memory. Nature 427: 605 .

Rife D.C. 1940. Handedness, with special reference to twins. Genetics 28: 178.

Schwarzacher H.G. and Schnedl W. 1966. Position of labelled chromatids in diplochromosomes of endo-reduplicated cells after uptake of tritiated thymidine. Nature 209: 107.

Segurado R., Detera-Wadleigh S.D., Levinson D.F., Lewis C.M., Gill J.I., Nurnberger J.I., Jr., Craddock N., DePaulo J.R., Baron M., Gershon E.S., et al. 2003. Genome scan metaanalysis of schizophrenia and bipolar disorder. III. Bipolar disorder. Am. J. Hum. Genet. 73: 49.

Vengrova S. and Dalgaard J.Z. 2004. RNase-sensitive DNA modification(s) initiates $S$. pombe mating-type switching. Genes Dev. 18: 794.

Watson J.D. and Crick F.H.C. 1953. Molecular structure of nucleic acids. Nature 171: 737. 


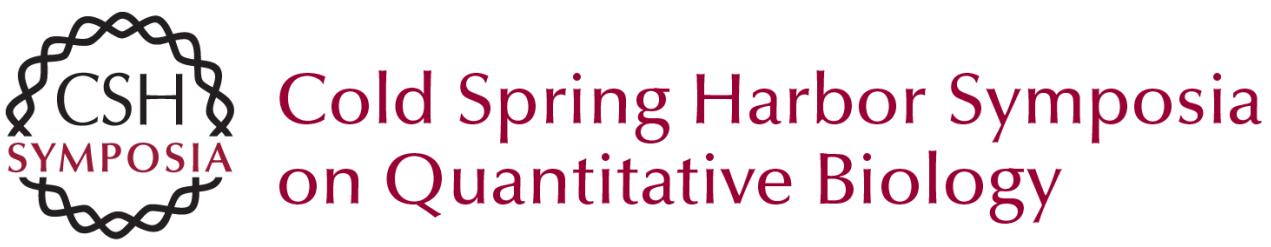

\section{An Epigenetic Hypothesis for Human Brain Laterality, Handedness, and Psychosis Development}

A.J.S. KLAR

Cold Spring Harb Symp Quant Biol 2004 69: 499-506

Access the most recent version at doi:10.1101/sqb.2004.69.499

References This article cites 35 articles, 12 of which can be accessed free at: http://symposium.cshlp.org/content/69/499.full.html\#ref-list-1

License

Email Alerting Receive free email alerts when new articles cite this article - sign up in Service the box at the top right corner of the article or click here. 